\title{
Téoros
}

Revue de recherche en tourisme

\section{Les défis environnementaux et culturels des stations de montagne}

\section{Une approche à partir du cas français}

\section{Philippe Bourdeau}

Volume 27, numéro 2, été 2008

Les grands équipements touristiques

URI : https://id.erudit.org/iderudit/1070794ar

DOI : https://doi.org/10.7202/1070794ar

Aller au sommaire du numéro

Éditeur(s)

Université du Québec à Montréal

ISSN

0712-8657 (imprimé)

1923-2705 (numérique)

Découvrir la revue

Citer cet article

Bourdeau, P. (2008). Les défis environnementaux et culturels des stations de montagne : une approche à partir du cas français. Téoros, 27(2), 23-30.

https://doi.org/10.7202/1070794ar d'utilisation que vous pouvez consulter en ligne. 


\section{Les défis environnementaux et culturels des stations de montagne Une approche à partir du cas français}

\section{Philippe Bourdeau}

Depuis la fin des années 1980, I'univers du tourisme de montagne européen est parcouru par de nombreux incertitudes et facteurs de changement structurels sectoriels ou globaux : stagnation ou érosion de la pratique du ski, perte de parts de marché touristique des pays alpins, accentuation de la concurrence entre destinations touristiques, émergence de nouvelles pratiques récréatives, vieillissement de la population... Le changement climatique, dont les effets sont d'ores et déjà fortement ressentis dans la vie touristique alpine, constitue un révélateur et souvent un accélérateur des mutations en cours. Pour de nombreux observateurs, le système des sports d'hiver hérité de la seconde moitié $d u X X$ siècle repose désormais sur un modèle épuisé, "qui a fait ses preuves et son temps " (Knafou, 1991), et qui est voué à une profonde recomposition impliquant de drastiques adaptations. À l'aune de ce changement et des enjeux du développement durable, le modèle porté par les plus grandes stations, s'il continue à s'afficher comme une réussite, n'est pas exempt de contradictions et de risques, avérés ou potentiels, que cet article se propose de questionner sous l'angle d'une approche géoculturelle du tourisme. Les stations françaises, qui font partie du trio de tête mondial ${ }^{1}$ de l'offre et de la fréquentation des sports d'hiver, fournissent à cet égard la matière d'une étude de cas d'autant plus propice qu'elles font la part belle à de grandes infrastructures touristiques, tant en nombre de lits touristiques qu'en nombre de remontées mécaniques et de pistes.

\section{La montagne : dernière frontière ou entre-deux ville nature?}

L'imaginaire de la montagne, tel qu'il est véhiculé dans la littérature, les médias et la publicité, est largement marqué par des images et des valeurs de liberté, de régénérescence physique et psychologique, d'affranchissement des normes sociétales. Comme d'autres grands espaces (la mer, les déserts), la montagne est donc représentée comme un " antimonde », un lieu de rupture utopique et uchronique par rapport aux contraintes associées à l'univers quotidien urbain (Bourdeau, 2007b). Mais l'idéal d'une montagne virginale et sauvage est contredit par la puissance de l'urbanisation, de l'aménagement et de l'omniprésence des médiations technologiques ou informationnelles qui ont accompagné la croissance de la fréquentation touristique de masse : (auto)routes, ensembles résidentiels, réseaux de remontées mécaniques et de canons à neige, portillons d'accès, signalétiques, réglementations... Ce qui n'empêche pas que la mythologie de la pureté et de la naturalité soit toujours convoquée pour promouvoir des aménagements et des équipements nouveaux.

Cette contradiction très classique, relevée par de nombreux auteurs, n'a cessé de s'accentuer depuis cinquante ans, en illustrant un des nombreux paradoxes de l'usage récréatif des espaces naturels, à savoir une tension majeure entre la culture et la technologie et la déclinaison de leurs diverses figures : le stylo qui raconte et la rou(t)e qui donne accès (Bertho-Lavenir, 1999) ; Icare le contemplatif et Prométhée le conquérant (Bozonnet, 1992)... De fait, cette dialectique fondatrice semble avoir pour enjeu le statut de la montagne dans les sociétés contemporaines :
" dernière frontière de la terre » sur le mode de la wilderness nord-américaine, selon une publicité Lafuma de 2004, ou bien entre-deux villemontagne mixant urbanisation dense et nature périphérique ou interstitielle, comme le suggère une publicité BMW de 2004, qui installe Paris au cœur d'un massif de haute montagne.

\section{Que reste t-il du " rêve blanc "?}

En Europe occidentale, le ski n'est plus aujourd'hui un indicateur d'ascension sociale comme dans les années 19601970. Fonctionnant sur un statut de tourisme " rituel » (Urbain, 1993), les sports d'hiver font de plus en plus figure de " niche » touristique élitiste, réservée à une clientèle internationale ou à des skieurs de proximité. Cette situation nourrit un sévère bilan critique, qui est accentué par les particularités de la situation française en comparaison avec des pays comme la Suisse et l'Autriche (Moreau, 2004), tant en ce qui concerne les options d'aménagement retenues que le statut très périphérique de la montagne et du ski :

- La logique financière et immobilière prime sur la logique sportive et culturelle dans un contexte de forte standardisation urbanistique et touristique de l'offre, même si l'« oubli » du skieur-client constaté dans les années 1980 cède la place à des démarches orientées vers la qualité.

- Du fait de leur gigantisme, les stations et les domaines skiables interconnectés sont des entités spatiales de plus en plus complexes et de moins en moins lisibles par les usagers, malgré la création de plans des pistes interactifs ou thématiques. 


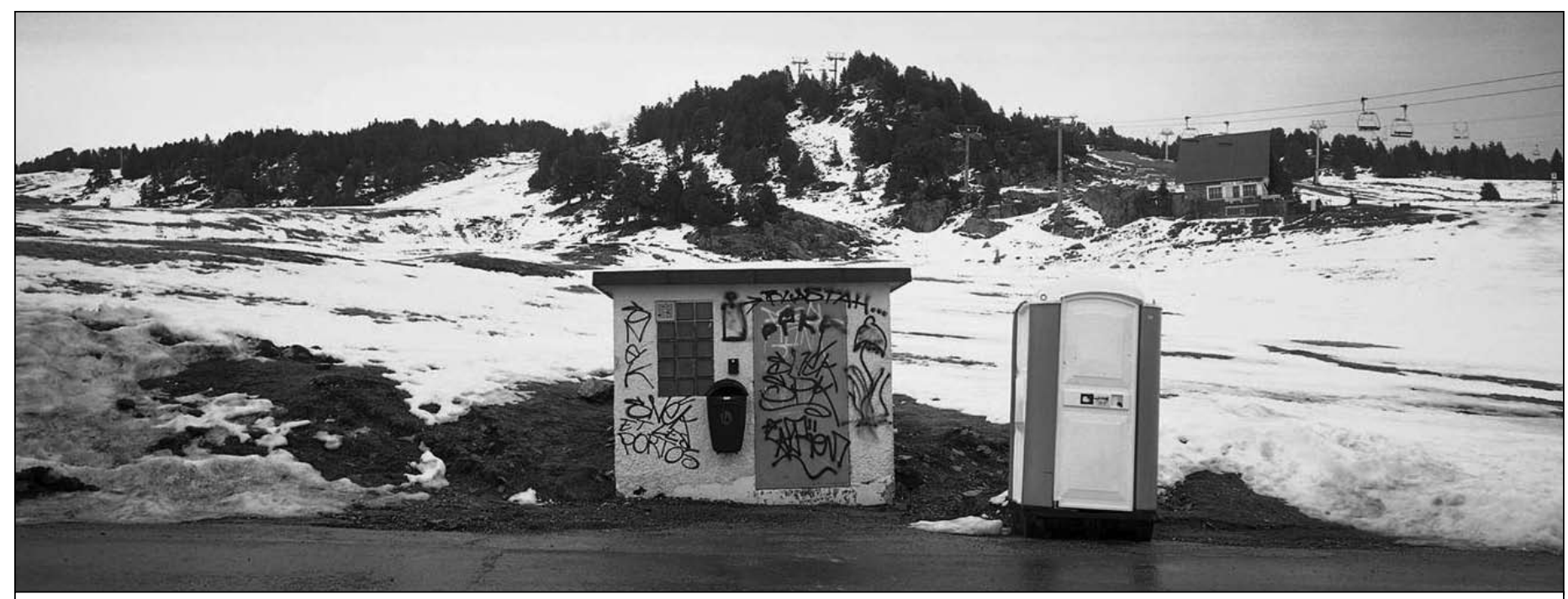

Illustration 1 : Station de Chamrousse (Isère, France) durant l'hiver 2006-2007.

Photo : Fred Pontoire.

- La stratégie de croissance en valeur (montée en gamme, augmentation du prix des forfaits...) privilégiée par les opérateurs touristiques (sur une croissance en volume de fréquentation) accentue le caractère élitiste des sports d'hiver et l'effet de niche de clientèle qui en résulte. Le ski est ainsi pratiqué chaque année par moins de $8 \%$ des français, et de 2 à $3 \%$ des adeptes " consomment " 70 à $80 \%$ des journées-skieurs (Berlioz, 2006). Dans ce contexte, les opérateurs concernés (privés ou publics) semblent avoir renoncé à la "démocratisation " du tourisme de montagne, ce que confirme l' " expulsion » de fait des différentes formes de tourisme social (colonies, camps, classes de neige) de la plupart des grandes stations par la pression foncière et immobilière.

- La banalisation et l'« usure » du ski traditionnel, pratiqué sur des pistes de plus en plus lissées, élargies (et parfois de ce fait plus dangereuses), est souvent pointée comme une source d'ennuis des skieurs, ce qui explique en partie la tendance à une baisse de la fréquence et de l'intensité de la pratique.

- En cas de déficit d'enneigement, comme durant l'hiver 2006-2007, le report des skieurs des stations privées de neige vers les stations les plus hautes semble se faire de moins en moins bien, à la grande inquiétude des professionnels les plus attentifs.

- Le parc de remontées mécaniques (plus de 3800 appareils) est considéré comme passablement vieilli et fait l'objet d'un rythme de renouvellement insuffisant, surtout dans les stations de moyenne montagne (Berlioz, 2006) ; dans de nombreux cas, les travaux réalisés sollicitent lourdement la contribution financière répétée des collectivités territoriales.

Dans ce contexte, la question iconoclaste " pourquoi les sports d'hiver? ", posée il y a trente ans par Guérin et Gumuchian (1978), ne manque pas de se voir posée à nouveau, notamment sous la forme de critiques radicales. Si toutes ne vont pas jusqu'à proclamer " démontons les stations! " en dénonçant leur inutilité sociale et leur nocivité écologique (Cheynet, 2004), ces positions sont régulièrement relancées par de multiples tensions et conflits d'ordre environnemental - extensions et liaisons entre domaines, programmes immobiliers, loisirs motorisés, gestion des ressources en eau ou à caractère socioéconomique - soutien financier des collectivités publiques, inégalités sociales d'accès aux sports de neige, statut et hébergement des travailleurs saisonniers. Depuis février 2004, date historique du premier mot d'ordre de grève lancé en France dans le secteur des remontées mécaniques, les mouvements sociaux liés à la question du travail saisonnier semblent destinés à se banaliser, en même temps que le tourisme devient une nouvelle terre de mission pour les grandes organisations syndicales. En effet, la forte augmentation des emplois dans le secteur des remontées mécaniques constatée entre 1985-1986 et 2002-2003 (de 12000 à 22 033, soit +84 \%) s'est accompagnée d'une diminution sensible de la proportion d'emplois permanents par rapport aux emplois saisonniers, qui est passée de $25 \%$ à $19 \%$. De même, les affres de la mondialisation commencent à être montrées du doigt jusque dans les stations les plus prestigieuses, où certaines arrière-cuisines sont réputées accueillir un personnel étranger en situation illégale, pendant que des garages serviraient de logement saisonnier à des moniteurs de ski originaires d'Europe de l'Est...

Il faut évidemment s'interroger sur la lecture quasi permanente des sports d'hiver en termes de crise, qui est faite depuis plus de trente ans par les journalistes ou les chercheurs, et, plus récemment, de l'intérieur, par un nombre croissant d'opérateurs. Qu'elle soit perçue comme un bégaiement défaitiste ou au contraire comme une lucidité opiniâtre, la récurrence de cette rhétorique de crise est en effet une caractéristique marquante de la représentation sociale des sports d'hiver. De la « montagne colonisée » 
(Cognat, 1973) à " la neige empoisonnée » (Arnaud, 1975) ou au " ski en crise » (Di Ruzza et Gerbier, 1977), des "ravages tout schuss " au "ski sur la mauvaise pente " en passant par "l'industrie du ski, cancer des Alpes ${ }^{2}$, une littérature critique abondante prend le contre-pied de l'unanimisme convenu sur les bienfaits de l'or blanc. Sur fond de changement climatique et de fin du pétrole bon marché, alors que l'assise démographique et sociale des sports d'hiver se réduit et que leur géographie se replie sur un nombre restreint de sites, cette eschatologie du ski comme " tradition menacée » et du skieur comme « espèce en voie de disparition " ${ }^{3}$ tend indéniablement à se renforcer, au delà d'un flagrant effet de "marronnier » médiatique. Ce faisant, ce ne sont pas seulement les contradictions internes des sports d'hiver qui sont visées. Dans un contexte d'exacerbation des sensibilités environnementales, de montée d'une exigence d'évaluation des politiques publiques et de regain de critique sociale, les stations - et surtout les plus grandes - sont considérées comme emblématiques d'un processus de " privatisation des profits " et de " collectivisation des pertes " (Mountain Wilderness, 2004) au sein duquel se conjuguent une modernité contestée et un élitisme arrogant.

\section{L'environnement : un invité de dernière minute dans les stations?}

Les enjeux d'un tourisme "durable " ne se réduisent pas à une problématique d'ordre environnemental. Toutefois, dans le cas français, la prise de conscience que l'attraction et la compétitivité des destinations ne reposent pas seulement sur des facteurs d'ordre technique et économique (Leïdner, 2004), mais dépendent aussi de facteurs de qualité écologique et paysagère, a été très tardive et reste partielle. C'est en grande partie par le biais d'une réflexion à marche forcée sur la qualité touristique, dans le contexte de crise de fréquentation des années 19801990, qu'a émergé lentement la question environnementale. La relation entre dégradation paysagère et « altération des images touristiques » (Debarbieux, 1995) est notamment devenue explicite dans les enquêtes réalisées auprès du grand public (SEMA METRA, 1987) et dans les médias. Pourtant, la réaction des opérateurs du tourisme de montagne s'est limitée durant toute la décennie 1990 à une repentance architecturale et néo-patrimoniale d'ordre purement esthétique (Wozniak, 2006) : de la Haute-Savoie aux Alpes-Maritimes, les stations font leur lifting et se parent à marches forcées de "vrais-faux vieux chalets tyroliens " pendant que des bardages, pièces de charpente " à l'ancienne " et autres chenaux en cuivre recouvrent les façades et les toits des bâtiments existants.

Malgré les fortes pressions exercées par des associations et des organisations non-gouvernementales ${ }^{4}$, il faut encore attendre le milieu des années 2000 pour que la question environnementale acquière une véritable légitimité. Après une démarche expérimentale de sensibilisation collective à l'environnement mise en œuvre dans les Pyrénées (ADEME, 2003) - ce qui témoigne au passage d'un décentrement significatif par rapport aux grandes stations alpines -, les initiatives dans ce domaine vont se multiplier. En réaction aux questionnements citoyens et médiatiques de plus en plus systématiques sur le choix d'une station écologiquement correcte ${ }^{5}$, l'argumentation environnementale devient désormais une figure imposée et occupe une place croissante dans la communication des opérateurs de sports d'hiver. Grâce à des actions pionnières, bien qu'isolées, des partenariats se nouent progressivement entre ONG (organisations non gouvernementales) et stations, notamment le séminaire "Sensibiliser à l'environnement en station de ski ", organisé en 2005 aux Ménuires par le Réseau alpin d'éducation à l'environnement montagnard et l'association Demain Vivre aux Belleville (REEMA, 2005), ou encore le « Respect festival », organisé en 2006 par la station de ski Les Arcs et l'association Mountain Riders. Sur fond de déficit d'enneigement, l'hiver 2006-2007 prend ainsi des airs de " temps de la révélation ", non seulement dans le domaine des rencontres et des événements consacrés à la question environnementale (premier forum international « Les stations s'engagent pour la planète " à Avoriaz, les " Ateliers de la terre " à Courchevel...), mais aussi dans le domaine de l'approvisionnement en énergie renouvelable. Plusieurs contrats de fourniture d'électricité verte sont ainsi signés entre des stations (Chamrousse, Serre-Chevalier) et des industriels producteurs d'énergie (Électricité de
France, Electrabel). Dans le même temps, sur les traces des Ménuires et de Montgenèvre, de plus en plus de stations entreprennent des démarches de certification environnementale (ISO 14001 et Qualité Sécurité Environnement) des remontées mécaniques et, plus récemment, réalisent des bilans d'émissions de gaz à effet de serre, même si elles ne s'engagent pas encore dans des mesures compensatoires, comme c'est le cas notamment à Aspen (États-Unis) ou à Arosa (Suisse). Par ailleurs, ce type d'initiative est loin d'atteindre en France le caractère systématique et collectif des démarches conduites depuis 2000 aux États-Unis autour de la charte Sustainable Slopes de la National Ski Areas Association (NSAA), qui engage près de 200 stations dans une réduction de leurs impacts environnementaux et climatiques (approvisionnement en énergies renouvelables, architecture de haute-qualité environnementale...) en complément d'une campagne d'information grand public intitulée Keep Winter $\mathrm{Coo}^{\prime}$. Mais il faut rappeler que les Golden Eagle Awards for Environmental Excellence ont été créés aux États-Unis dès 1993 pour reconnaitre les efforts d'intégration environnementale des stations de ski...

Dans les stations françaises, les efforts réalisés se limitent pour l'instant, dans la plupart des cas, à des actions très basiques et surtout très ponctuelles : sensibilisation environnementale du personnel et de la clientèle, mise en place de poubelles « intégrées au paysage ", tri sélectif des déchets, distribution de cendriers de poche, procédures de prévention de pollutions diverses de l'air et de l'eau... À tel point que la crédibilité même de ces démarches est souvent mise en cause comme une simple " poudre aux yeux " ${ }^{7}$. Les travaux entrepris sur les pistes de ski intègrent néanmoins des contraintes de plus en plus lourdes, souvent liées à des conflits avec des groupes de pression vigilants et les gestionnaires d'espaces protégés (Marcelpoil et Langlois, 2006) : notices et études d'impacts systématiques, allongement des délais et de la durée des travaux, surcoûts conséquents.

S'il semble aussi général que tardif, le consensus sur les exigences de qualité environnementale des sports d'hiver n'est cependant pas exempt de contradictions majeures dont 
les plus épineuses peuvent être désignées comme le syndrome «AIE ! (AutomobileImmobilier-Enneigement artificiel).

- L'automobile, vecteur majeur de la massification du tourisme et moyen d'accès privilégié aux stations pour plus de $80 \%$ des nuitées (SEATM, 2008), est en montagne comme ailleurs une source paradoxale de liberté de mouvement, mais aussi de contraintes et d'impacts. Au-delà de la question classique de la saturation saisonnière des accès routiers aux stations, la problématique de la place de l'automobile au sein de celles-ci (circulation, stationnement, bruit, pollution, relation avec les piétons...) est aujourd'hui un véritable défi, en été comme en hiver. II faut pourtant convenir à ce sujet que, malgré quelques expériences précoces de stations sans voitures (Avoriaz, Flaine), les stations françaises sont en retrait par rapport à la généralisation d'initiatives de mobilité douce dans des stations allemandes, autrichiennes, italiennes et suisses. Aucune d'entre elles n'a ainsi participé au projet Alps Mobility I (Programme Interreg IIc 1998-2001) et une seule - la petite station des Gets, en Haute-Savoie - a participé à son second volet 2003-2006. De même, aucune destination ou organisation française ne figure parmi la vingtaine de partenaires du Network for Soft Mobility in European Tourism (NETS) ${ }^{8}$ et les principales initiatives notables sur ce registre restent encore et toujours le fait des ONG. Le bilan carbone pionnier réalisé par l'association Mountain Riders dans les stations des Ménuires et de Val Thorens indique pourtant que $74 \%$ des gaz à effet de serre émis sont imputables au transport des skieurs ${ }^{9} .$.

- Le deuxième " moteur et frein " du tourisme d'hiver dans les montagnes françaises est l'immobilier. II est couramment admis par les observateurs et les opérateurs concernés que, depuis quarante ans, la construction immobilière joue un rôle de financement externe des investissements dans les remontées mécaniques (Berlioz, 2006). Alors que la pratique du ski tend à concerner une population de plus en plus restreinte, environ 20000 lits nouveaux sont construits chaque année dans les Alpes du nord et le découplage entre fréquentation sportive et marché immobilier ne cesse de s'accentuer, en bénéficiant de mesures d'incitation fiscale. Celles-ci souffrent souvent d'effets pervers, comme le montre l'exemple du dispositif des Zones de revitalisation rurale (ZRR) initié en 1995, dont plus de la moitié des investissements pour la période 2000-2002 se sont concentrés sur six communes de Maurienne, alors que 20000 communes françaises étaient concernées (Mountain Wilderness, 2004). De fait, au delà des stratégies commerciales propres aux constructeurs, le problème des gestionnaires de stations est de compenser la sortie de nombreux lits du marché locatif, du fait de l'obsolescence ou de stratégies spéculatives. Cette fuite en avant immobilière se poursuit, alors même que l'image des stations françaises à l'étranger est de plus en plus altérée par une urbanisation excessive, dont la régulation politique et urbanistique suscite des polémiques récurrentes ${ }^{10}$.

- L'enneigement artificiel est la dernière solution-problème du triptyque "AlE ». Quasi unique palliatif choisi face au déficit de neige naturelle, il apparait aussi de plus en plus comme une source croissante d'impacts environnementaux et socioéconomiques désormais relevés par la plupart des observateurs (CIPRA, 2004 ; 2006 ; OCDE, 2007) : artificialisation accentuée des domaines skiables du fait de travaux d'installation d'enneigeurs et de reprofilage des pistes, conséquences sur la biodiversité, surconsommation d'énergie et d'eau ${ }^{11}$. La question de l'approvisionnement en eau des équipements d'enneigement, très médiatique et conflictuelle, est particulièrement emblématique de la globalité des problèmes posés, qu'il s'agisse de la modification du régime des eaux des torrents et de la réduction de biodiversité qui en résulte, de la construction et de l'approvisionnement des retenues collinaires de plus en plus nombreuses et de plus en plus vastes, de la concurrence avec l'approvisionnement en eau potable et de la perte d'eau par évaporation. Mais les conditions techniques de production artificielle de neige sont aussi de plus en plus problématiques : emploi éventuel d'additifs bactériologiques ou chimiques, mise en œuvre de dispositifs réfrigérants, recours à un transport routier ou héliporté de neige de culture ${ }^{12}$. En outre, alors que son objectif initial était de pallier le déficit d'enneigement à basse altitude, l'enneigement artificiel est de plus en plus utilisé en haute altitude, y compris sur glacier, notamment dans le but de prolonger les saisons de ski.

Face à ce cumul de handicaps structurels et fonctionnels, le recours à des solutions de " communication verte " (publicité, labellisations autoproclamées et jamais évaluées, certifications peu ambitieuses malgré les effets d'annonce...) est fréquemment observé. «Val Thorens vire au vert » annonce ainsi en février 2008 un journal régional ${ }^{13}$ au sujet d'une des grandes stations de Tarentaise les plus emblématiques des contradictions environnementales fondatrices de l'exploitation de l'or blanc. On citera encore l'édition française récente de la revue Onboard ${ }^{14}$, qui affiche sur sa couverture une surenchère écologiquement correcte ("Le numéro 100 \% vert ", "The eco-issue ", « degradable plastics »...), mais révèle en quatrième de couverture un snowboardeur radieux posant devant un magnifique hélicoptère! Cette "schizophrénie » semble inhérente au registre du " tourisme durable " en tant que point maximal de critique possible dans les limites du modèle idéologique et économique dominant. On notera malgré tout l'élévation significative du niveau d'exigences du public, des médias et des opérateurs eux-mêmes, ces derniers se retrouvant à moyen terme de plus en plus pris au jeu - et parfois pris au piège - des positions volontaristes qu'ils affichent.

\section{Y a-t-il une vie (récréative) en dehors des stations?}

Une lecture des revues spécialisées des hivers 2006-2007 et 2007-2008 ${ }^{15}$ relève sans surprise la permanence des icônes et des slogans riches en promesses de neige poudreuse et de sensations, tout comme elle confirme la vitalité d'une sous-culture de la glisse... Mais un examen plus approfondi révèle une fragmentation géoculturelle croissante entre les figures de la glisse urbaine (wall-ride, omniprésence des références à la ville et aux structures industrielles) et les figures du voyage et des "spots " exotiques (Cachemire, Kamtchatka, Colombie-Britannique, Australie...), où les mythologies de la glisse et du nomadisme sont célébrées sur un mode qui oscille entre 


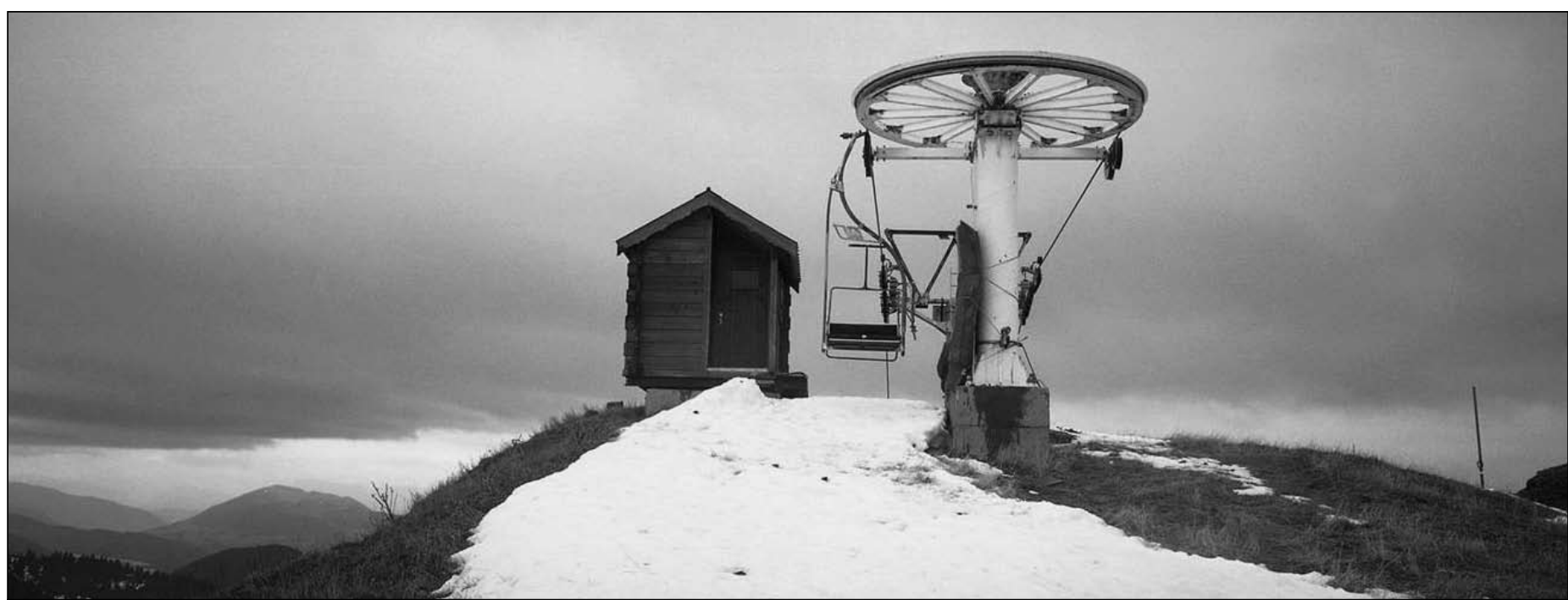

Illustration 2 : Remontée mécanique à Chamrousse (Isère, France) durant I'hiver 2006-2007.

Photo : Fred Pontoire.

la contre-culture classique et la provocation ludique. À l'exception des quelques espaces publicitaires qui leur sont dédiés, les stations de sports d'hiver semblent en revanche largement inscrites en creux dans ce paysage médiatique. Cet effacement se manifeste par la prééminence des marques de matériel dans les contenus publicitaires et rédactionnels, comme dans la définition de l'appartenance des sportifs de haut niveau, lesquels sont désormais associés aux industriels et non plus aux stations. Mais il se lit aussi de plus en plus dans les déclinaisons communautaires des événements sportifs en fonction du genre, à l'image du Rip Curl Girls Tour qui parcourt douze stations européennes, ou encore dans les dynamiques d'hybridations sportives et festives d'intérieur, comme l'Air \& Style qui réunit sur la même scène planche à neige, motocross et concert musical à Munich, ou l'Imperium \& Technine Harbour Rail Jam organisé dans le port d'Anvers. Bien sûr, chaque revue spécialisée offre un point de vue très partiel sur la culture récréative à laquelle elle se réfère et, en élargissant le corpus à d'autres titres $^{16}$, on accède à une image classique dans laquelle les stations gardent un certain droit de cité. Mais cette esquisse de revue de presse confirme, s'il en est besoin, que le climat n'est pas la seule variable de mutation de l'univers des sports d'hiver. Un changement de mythologie touristique s'opère également et introduit dans les stations, mais aussi entre elles et en dehors d'elles, de nouveaux usages de l'espace et de nouvelles formes de pratique des sports de neige. En témoignent par exemple les multiples raids, camps et circuits itinérants qui se jouent de l'univers statique des stations tout en s'appuyant en partie sur elles.

La notion de station comme unité idéale de lieu, de temps et d'action, basée sur la cohérence fonctionnelle du triptyque hébergement / remontées mécaniques / pistes, peut ainsi se trouver contournée ou détournée par de nouvelles lectures du terrain de jeux montagnard et les usages inattendus qui en découlent. C'est par exemple le cas quand les utilisateurs d'un grand domaine skiable privilégient des points d'entrée secondaires dans celui-ci pour des raisons d'ambiance et de qualité. De même, un contraste saisissant peut être observé entre la course au gigantisme dans l'extension et l'interconnexion des domaines skiables et la micro-échelle spatiale à laquelle se situent les pratiques émergentes des jeunes glisseurs : le module d'un snowpark (parc de planche à neige), mais aussi de plus en plus une banale pente, une bosse " améliorée », un rocher, un tronc d'arbre, un escalier enneigé ou une porte de bâtiment, deviennent les supports possibles d'une expression centrée sur la gestuelle, le jeu et le partage des émotions, dans laquelle le façonnage et le bricolage
" sur mesure " du terrain avec des outils simples (mains, pelle, planche à neige...) prennent une place à part entière, alors que le recours aux remontées mécaniques devient accessoire. Ces jeux sélectifs ou alternatifs avec les ressources standardisées offertes par les stations, qui favorisent la proximité, la simplicité, l'interstice ou l'écart - et s'accommodent volontiers d'un enneigement déficient -, se retrouvent aussi dans les aspirations de beaucoup de visiteurs à sortir des pistes ou à chercher entre celles-ci des terrains d'expression ludique renouvelés, à skis, en raquettes à neige ou à pied. Ce faisant, ce n'est plus seulement la spatialité des pratiques qui s'en trouve modifiée, mais aussi leur temporalité et leur sens, puisque l'objectif n'est plus d'enchaîner descentes et remontées, mais au contraire de faire des détours aux allures de jeu de piste, voire des pauses, dans l'exploration buissonnière d'un domaine skiable et de ses environs.

Même si dix opérateurs, dont huit situés en Tarentaise, concentrent $40 \%$ du chiffre d'affaires des remontées mécaniques de l'ensemble des stations françaises, c'est un scénario de diversité et de cohabitation entre différentes logiques de destinations touristiques hivernales qui prévaut plus que jamais. Une typologie provisoire peut ainsi être esquissée autour de six principales catégories de lieux récréatifs : 
1. grandes et moyennes stations économiquement " compétitives » gérées par des opérateurs industriels ;

2. stations moyennes dépendantes des voyagistes et des aides publiques, confrontées à des situations de crise récurrentes et à des processus de reconversion difficiles ;

3. petites stations fonctionnant sur le modèle " culturel et territorial », orientées vers une forte diversification saisonnière et économique et fréquemment engagées dans une reconversion résidentielle ;

4. micro-stations " compétitives 》 gérées selon le modèle industriel par de petits opérateurs, éventuellement dans le giron de grands groupes ;

5. stades de neige ou micro-stations traditionnelles et/ou " underground ", situés à proximité des grandes agglomérations alpines dans une logique de fréquentation intermittente en fonction de l'enneigement, à la (demi-)journée, voire à la soirée.

6. sites, «spots » et itinéraires de pratique fréquentés hors de toute logique de station.

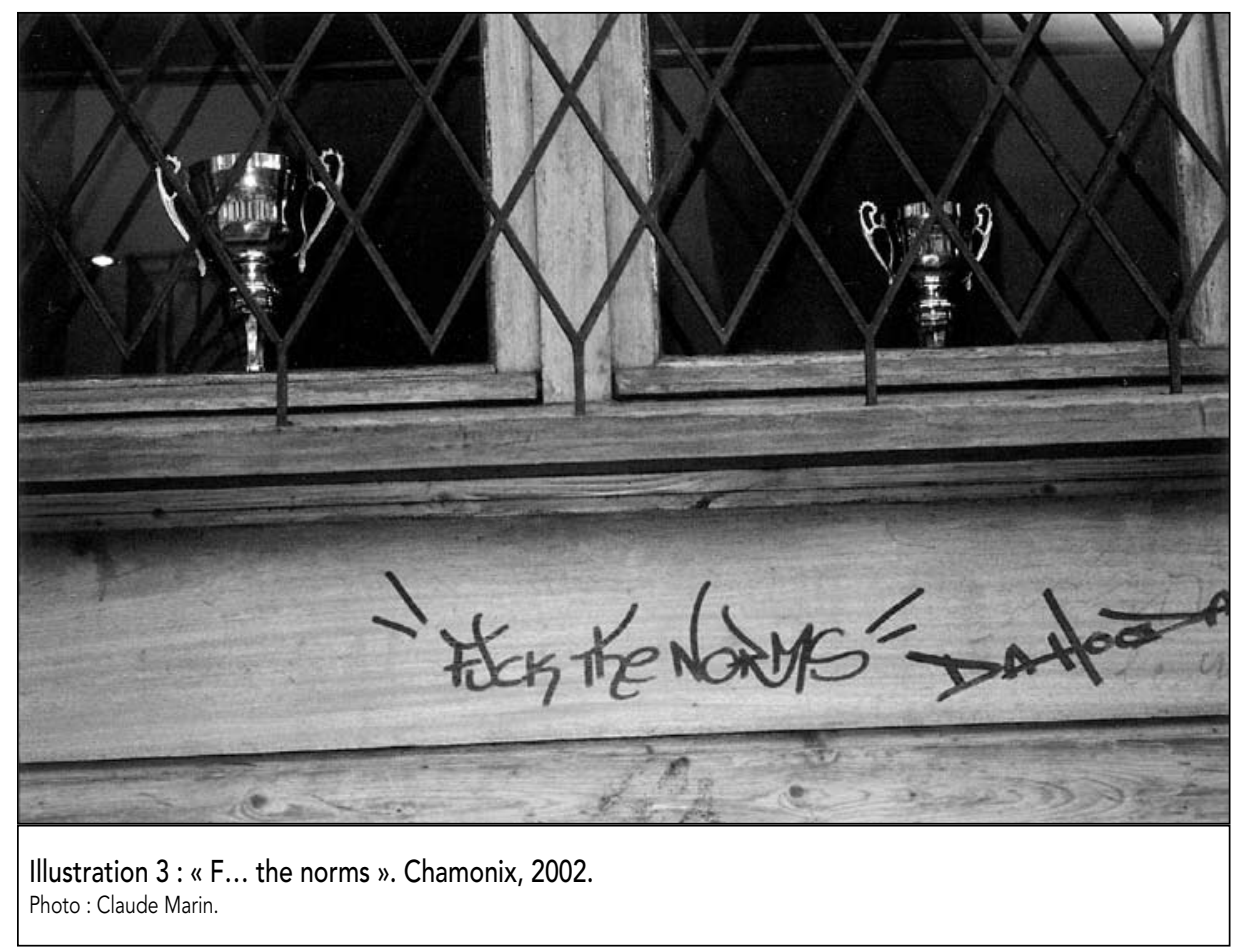

\section{De l'aménagement à la culture : un changement de paradigme ?}

L'évidence structurelle croissante de l'« industrie touristique » alpine s'impose par l'ampleur des réalisations qui rythment l'espace et le temps des grandes stations : promotion immobilière, téléphériques géants, aquadômes... Bien entendu, la création et la gestion des stations selon des règles techniques et économiques de rationalité et d'efficacité est présente dès les années 1960 et 1970, notamment en France. Mais c'est avec la montée en puissance d'enjeux de compétitivité et de qualité, avec le poids de plus en plus fort de la clientèle internationale, ou encore l'inflation des investissements dans la neige de culture ou les remontées mécaniques dans les années 1990 et 2000, que le tourisme alpin s'est largement industrialisé ; tout comme les restructurations et les concentrations financières se sont accélérées à l'échelle régionale et mondiale avec le développement de grands opérateurs, par exemple la Compagnie des Alpes et Intrawest.

Pourtant, au-delà des facilités de langage et de la volonté de légitimer le caractère " sérieux » de l'activité touristique, les limites du modèle touristique industriel comme horizon de référence sont bien connues depuis trente ans :

- Il concerne une minorité de stations, même s'il s'agit des plus fréquentées. En France, par exemple, moins de 30 stations sur plus de 300 centres de ski alpin, soit à peine $10 \%$ des domaines skiables recensés, y participent, à des degrés divers, par le biais de l'intervention d'opérateurs comme la Compagnie des Alpes, SEM 3 Vallées, SOFIVAL, SATA, Transmontagne, Rémy Loisirs... De plus, il reste très fortement dépendant de la saison d'hiver, malgré les enjeux de développement d'un «tourisme des quatre saisons » en montagne.

- Il est critiqué pour sa standardisation de l'offre et son incapacité chronique à s'adapter à l'évolution de la demande ; la fin de l'« équation touristique du premier degré : sports d'hiver = unicité (ski) + uniformité (stations) + répétitivité (séjour annuel) »(Chevalier, 1996) semble ainsi être une rupture particulièrement difficile à comprendre et à prendre en compte par ses opérateurs. Cette rigidité l'a déjà rendu sujet à de profondes crises depuis 20 ans : crise des sports d'hiver en France (fin des années 1980), crise du tourisme balnéaire aux Baléares (début des années 1990).

Dans la pratique, la conception habituelle de la station comme " usine à rêves » tourne le plus souvent au cauchemar, ce que constate un éditorial récent de la revue professionnelle Montagne Leaders intitulé « La montagne ne fait plus rêver ${ }^{17}$. Par une ironie sans doute involontaire, cet éditorial très lucide est accompagné de photographies qui illustrent fort bien les dérives industrielles du tourisme de montagne : des paysages envahis de remontées mécaniques, un loisir artificiel et superficiel (dévalkart), sans oublier le portrait amène d'un des promoteurs immobiliers français les plus contestés des trente dernières années...

Face à l'évidence du modèle « industriel » du tourisme, la notion de modèle culturel et territorial fait pourtant figure de parent pauvre. La question culturelle est même la plupart du temps éludée en tant que variable-clé du renouvellement du sens de la pratique 


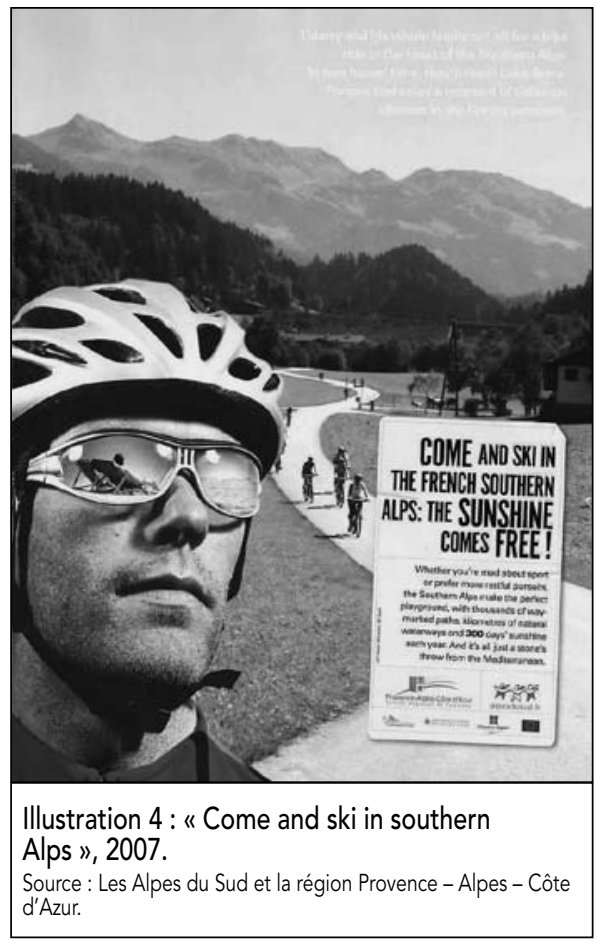

touristique en montagne, celle-ci n'étant plus considérée que comme un phénomène purement consommatoire, apparemment orphelin de rêves et de modèles autres que ceux de la distinction sociale et du placement immobilier. L'innovation touristique n'en mérite pas moins une attention renouvelée repensée sous l'angle de l'autonomie culturelle (Bellefleur, 2002) : réfléchir en termes de pratiques et d'expériences et non seulement en termes d'équipement et de consommation pour aborder la demande touristique ; (re)donner de l'importance aux processus non marchands comme pratiques sociales et facteurs de créativité ; poser la question des limites (définition, gestion...) dans la normalisation et la standardisation des espaces récréatifs...

À ce titre, la conception dominante d'une innovation portée avant tout par l'intervention de l'État, du mouvement sportif institutionnalisé ou des acteurs économiques semble peu réaliste tant la dynamique socioéconomique liée au tourisme peut être considérée comme dérivée de la culture, et non l'inverse. L'histoire du tourisme et des loisirs sportifs illustre ce processus en reposant pour une part essentielle sur des détournements, des retournements et des contournements créatifs liés à des pratiques buissonnières, marginales, improbables : bricolages et expérimentations (parapente, hydrospeed / luge à eau, snowboard / planche à neige, vélo toutterrain), relances et réinventions (télémark, raquettes à neige, luge, snow-kite), transgressions (sauts de routes, remontées au clair de lune, bivouac)...

\section{Conclusion Les grandes stations de sports d'hiver - entre anciennes ren- tes et nouvelles exigences}

"Et pourtant elles tournent ! » pourrait s'étonner à juste titre un Tintin au pays de l'or blanc à propos des stations de ski. De fait, on ne peut perdre de vue que le "système des sports d'hiver » fonctionne bel et bien et ce, malgré les critiques et les diagnostics catastrophistes dont il fait l'objet depuis trois décennies. Mais les conditions mêmes de cette fonctionnalité apparaissent de plus en plus paradoxales, comme l'illustre le cas français. Tout en bénéficiant de notables avantages structurels et conjoncturels (chiffres d'affaires en hausse, fréquentation internationale, altitude souvent élevée garantissant un enneigement naturel dans les prochaines décennies, capacité d'investissement dans la neige de culture, image de marque...), les grandes infrastructures touristiques de montagne semblent toujours face à une profonde évaluation de leur mode de développement. Cette interpellation pourrait paraître très rhétorique si elle ne reposait pas sur un ensemble de questions dont l'acuité se renforce, qu'il s'agisse de la gestion d'un encombrant héritage paysager et urbanistique, de la pleine appropriation d'une responsabilité environnementale, de la gouvernance territoriale (Gerbaux et Marcelpoil, 2006) ou de la relance du sens et de la qualité de l'expérience vécue par les visiteurs (Bourdeau, 2007a). Alors que les données disponibles confirment que le tourisme d'été représente pour la montagne française - dans toute sa diversité géographique et socioéconomique - un chiffre d'affaires supérieur à celui du tourisme d'hiver, les stations sont confrontées à un enjeu d'ancrage dans leur territoire support (Gaïdo, 2002 ; Corneloup, 2007 ; Marcelpoil, 2007) pour éviter de se voir réduites à une fonction de comptoirs touristiques. Enfin, alors que la plupart d'entre elles se définissent d'abord comme "stations de sports d'hiver », elles ne peuvent plus faire l'économie de l'élaboration d'une vision de l'avenir dans laquelle la place du ski, de la neige, de la saison hivernale, voire du tourisme (ANEM, 2007), doit être repensée. Si le changement climatique (Bürki et al., 2005) constitue à cet égard un facteur de mutation majeur, il n'est pas le seul : les migrations d'agrément (Moss, 2006) et la notion émergente d'économie « présentielle » (Davezies, 2008) renouvellent singulièrement la vision classique de l'économie touristique. C'est dans ce contexte que de nouveaux enjeux d'observation se font jour pour comprendre et accompagner les scénarios d'adaptation, de diversification ou de crise dans lesquels s'engageront les stations de montagne.

Philippe Bourdeau est professeur à l'Institut de Géographie Alpine de l'Université Joseph Fourier (Grenoble I).

\section{Notes}

1 Avec leurs homologues des États-Unis et d'Autriche.

2 Références dans l'ordre de citation : Article dans Libération du 18 décembre 2003 ; Journal du CNRS, janvier 2006, Dossier " Aménagement en montagne/Industrie du ski ", [www.moutain-wilderness.org], consulté le $1^{\text {er }}$ décembre 2005.

3 Références dans l'ordre de citation Environnement, revue de l'Office fédéral suisse de l'environnement, $n^{\circ} 2$ 2, 2003, p. 16 ; [www.cipra.org], brève du 23 mars 2006.

4 Les plus actives sont : la Commission internationale pour la protection des Alpes, Mountain Wilderness, le Réseau alpin d'éducation à l'environnement montagnard et Mountain Riders.

5 Par exemple le Guide vert des stations de montagne 2006-2007 édité par l'association Mountain Riders, [www.mountain-riders.org], et Le Monde, 23 novembre 2006.

6 [www.nsaa.org/nsaa/environment/sustainable_slopes] et [http://keepwintercool.org].

7 Voir le Bulletin été 2005 de Mountain Wilderness : " Les stations cherchent à s'acheter une bonne conduite environnementale : un peu de revégétalisation et de la 'com' suffisent pour obtenir une certification ISO $14001 »$ (p. 25).

8 [www.soft-mobility.com].

9 [http://tinyurl.com/6dxjcu].

10 Voir par exemple " Haute Maurienne, la vallée verte deviendra grise ", Libération du 13 novembre 2006. 
11 D'après les données de l'ODIT [ODI 06] (Observation, développement et ingénierie touristiques), la puissance installée en France est de près de 210000 kilowatts et la consommation d'eau de 16 millions de mètres cubes en 2005-2006.

12 Citons, à titre de simples illustrations, I'utilisation de 1,5 tonne de nitrate d'ammonium pour la préparation d'une piste de compétition de slalom du Lauberhorn dans l'Oberland Bernois au début de janvier 2007 (Source : Alpmedia.net, 18 janvier 2007) ; ainsi que I'héliportage de 20 mètres cubes de neige de culture pour enneiger le bas d'une piste à Saint-Jean d'Aulps (Savoie) à la fin décembre 2006 (Source : Le Messager, 4 janvier 2007).

13 Supplément week-end du journal Le Progrès, 21 février 2008, p. 15.

14 Onboard, octobre 2006.

15 Notamment Snow surf et Skieur Magazine.

16 Par exemple : Ski magazine.

17 Montagne Leaders n²02, août 2007.

\section{Bibliographie}

Agence de l'Environnement et de la Maîtrise de l'Énergie (ADEME), Agence Régionale pour l'Environnement (ARPE), Service d'études et d'aménagement touristique de la montagne (SEATM) et Syndicat National des Téléphériques de France (SNTF) (2003), Guide de sensibilisation à l'environnement des stations de sports d'hiver pyrénéennes, Toulouse.

Arnaud, Danièle (1975), La neige empoisonnée, Paris, A. Moreau.

Association Nationale des Élus de la Montagne (ANEM) (2007), Au-delà du changement climatique, les défis de l'avenir de la montagne, Paris.

Bellefleur, Michel (2002), Le loisir contemporain. Essai de philosophie sociale, Sainte-Foy, Presses de l'Université du Québec.

Berlioz, Frédéric (2006), Les données de base de la connaissance : analyse des évolutions, impact sur le devenir du tourisme en montagne. Challes-Les-Eaux (France), Direction des études et de l'aménagement touristique de la montagne / Observation, développement et ingénierie touristiques (DEATM-ODIT).

Bertho-Lavenir, Catherine (1999), La roue et le stylo. Comment nous sommes devenus touristes ?, Paris, Odile Jacob.

Bourdeau, Philippe (2007a), « L'après-ski a commencé ", dans Philippe Bourdeau (dir.), Les sports d'hiver en mutation. Crise ou révolution géoculturelle ?, Cachan, HermèsLavoisier, p. 207-231.

Bourdeau, Philippe (2007b), "Les sports de nature comme médiateurs de l'entre-deux ville-montagne : vers un post-tourisme ? ", dans Lilli Monteventi-Weber, Chantal Descheneaux et Michèle Tranda-Pitton (dir.), Campagne-ville, le pas de deux. Enjeux et opportunités des recompositions territoriales, Lausanne, Presses polytechniques et universitaires romandes p. 27-36.

Bozonnet, Jean-Paul (1992), Des monts et des mythes. L'imaginaire social de la montagne, Grenoble, Presses universitaires de Grenoble.

Bürki Rolf, Elsasser Hans et Bruno Abegg (2005), "Climate Change and Tourism in the Swiss Alps ", dans Michael Hall et James Higman (dir.), Tourism, Recreation and Climate Change, Clevedon (UK), Channel View Publications, p. 155-163.

Chevalier, Marc (1996), «Paroles de modernités. Pour une relecture culturelle de la station de sports d'hiver moderne ", Revue de géographie alpine, n 3 , p. 29-39.

Cheynet, Vincent (2004), "Démontons les stations de sports d'hiver ", La Décroissance, $n^{\circ} 25$, p. 7 .

Cognat, Bruno (1973), La montagne colonisée, Paris, Le Cerf.

Commission Internationale pour la Protection des Alpes (CIPRA) (2004), L'enneigement artificiel dans l'arc alpin, rapport de synthèse, [Alpmedia.net].

Commission Internationale pour la Protection des Alpes (CIPRA) (2006), " Le tourisme d'hiver en mutation. Skier aujourd'hui et pour toujours, amen ? ", Cipra Info, no 81, numéro thématique.

Corneloup, Jean (2007), " Ambiance et univers culturels dans les stations de sports d'hiver ", dans Philippe Bourdeau (dir.), Les sports d'hiver en mutation. Crise ou révolution géoculturelle ? Cachan, Hermès-Lavoisier, p. 183-193.

Davezies, Laurent (2008), La république et ses territoires. La circulation invisible des richesses, Paris, Le Seuil.

Debarbieux, Bernard (1995), Tourisme et montagne, Paris, Economica.

Di Ruzza, François et Bernard Gerbier (1977), Ski en crise ; le grand cirque blanc : du profit à la compétition, Grenoble, Presses universitaires de Grenoble.

Gaïdo, Luigi (2002), « Du concept de station au concept de district ", Revue de géographie alpine, $n^{\circ} 4$, p. 109-115.

Gerbaux, Françoise et Emmanuelle Marcelpoil (2006), "Gouvernance des stations de montagne en France : les spécificités du partenariat public-privé ", Revue de géographie alpine, tome 94, no 1, p. 9-31.

Guérin, Jean-Paul et Hervé Gumuchian (1978), Pourquoi les sports d'hiver? Mythologies et pratiques, Grenoble, Institut de Géographie Alpine.
Knafou, Rémy (1991), « La crise du tourisme dans les montagnes françaises. Un système qui a fait ses preuves et son temps ", Dossier de la Revue de géographie alpine, nº 6, p. 13-21.

Leïdner, Rudiger (2004), The European Tourism Industry. A Multi-sector with Dynamic Markets. Structures, Developments and Importance for Europe's Economy, Luxembourg, Office for Official Publications of the European Commission.

Marcelpoil, Emmanuelle (2007), " L'ancrage territorial des stations de montagne ", dans Philippe Bourdeau (dir.), Les sports d'hiver en mutation. Crise ou révolution géoculturelle?, Cachan, Hermès-Lavoisier, p. 161-172.

Marcelpoil, Emmanuelle et Laurent Langlois (2006), "Protection de l'environnement et développement touristique en station : du conflit à l'organisation des proximités ", Revue durable et territoires, $n^{\circ} 7$, [http:// developpementdurable.revues.org/document2660.html], consulté le 16 mai 2008.

Moreau, Pierre (2004), Étude sur le positionnement de l'offre française de sports d'hiver, Rapport au Service d'études et d'aménagement touristique de la montagne (SEATM).

Moss, Laurence (dir.) (2006), The Amenity Migrants: Seeking and Sustaining Mountains and their Cultures, Wallingford, Cabi Publishing.

Mountain Wilderness (2004), La quête de l'or blanc, Bulletin n 61, Grenoble.

Organisation de coopération et de développement économiques (OCDE) (2007), Changement climatique dans les Alpes européennes: adapter le tourisme d'hiver et la gestion des risques naturels, OCDE, Synthèse de l'étude sur : [www.oecd.org].

Réseau alpin d'éducation à l'environnement montagnard (REEMA) (2005), Sensibiliser à l'environnement en station de ski, Compte-rendu du week-end d'échanges aux Ménuires, [http://reema.fr/client/projet/fichiers/S9/ S9_51.pdf], consulté le 16 mai 2008.

SEMA-METRA (1987). Étude des loisirs de montagne, les marchés des stations de sports d'hiver, 2 tomes, Paris, 1987-1988.

Service d'études et d'aménagement touristique de la montagne (SEATM) (2008), Chiffresclés du tourisme de montagne, [http:// www.odit-france.fr/Les_chiffres_cles_du_ tourisme.405.0.html], consulté le 16 mai 2008.

Urbain, Jean-Didier (1993), L'idiot du voyage. Histoires de touristes, Paris, Payot.

Wozniak, Marie (2006), L'architecture dans l'aventure des sports d'hiver. Stations de Tarentaise 1945-2000, Mémoires et Documents, L'Histoire en Savoie, Chambéry, Société savoisienne d'histoire et d'archéologie / Fondation pour l'action culturelle internationale en montagne (SSHA Facim). 